\title{
Klinische Studie zur Wirkung einer äusserlich angewendeten Zubereitung mit Glukosaminsulfat, Chondroitinsulfat und Kampfer bei Kniegelenkarthrose
}

Glukosamin- und Chondroitinsulfat sind Wirkstoffe mit geringer Toxizität, die Schmerz und Gelenksteife bei Arthrose reduzieren. Langfristige Anwendung von Glukosamin kann die Röntgenprogression von Arthrose reduzieren. Für Chondroitinsulfat wurde ein schmerzreduzierender Effekt nachgewiesen; zudem verstärkt es die Schmerzreduktion von Glukosamin.

Die Abteilung für Komplementärmedizin, RMIT University, und das Zentrum für Komplementärmedizin, Monash University, beide in Melbourne, Australien, untersuchten die Wirkung einer Salbe mit Glukosaminsulfat, Chondroitinsulfat und Kampfer bei Kniegelenkarthrose in einer monozentrischen, randomisierten Placebokontrollierten, doppelblinden Studie [1].

\section{Studie}

Die Probanden wurden in zwei Behandlungsgruppen mit ähnlicher demografischer Zusammensetzung und Krankheitsgeschichte eingeteilt. Teilnehmer: Patienten mit objektiv dokumentierter Kniegelenkarthrose (nach der Definition des American College of Rheumatology) sowie mit Knieschmerz, der mit $>4 \mathrm{~cm}$ auf einer $10 \mathrm{~cm}$ visuellen Analogskala (VAS) in einem oder beiden Knien für $>4$ Wochen bewertet wurde.

Die Patienten wurden randomisiert und erhielten eine äußerlich anzuwendende Glukosamin/Chondroitin-Zubereitung $(\mathrm{n}=32)$, oder Placebo $(\mathrm{n}=31)$, die 8 Wochen lang nach Bedarf angewendet wurde. Das Glukosamin/Chondroitin-Präparat war eine wasserlös- liche Creme mit 3,0 mg/g Glukosaminsulfat, 7,2 mg/g Chondroitinsulfat, $14 \mathrm{mg} / \mathrm{g}$ Haiknorpel (das zu 10-30\% aus Chondroitinsulfat besteht), $32 \mathrm{mg} / \mathrm{g}$ Kampfer, parfümiert mit Pfefferminzöl (9 mg/g). Die Placebo-Zubereitung war eine einfache kosmetische Creme mit gewöhnlichen Hautpflegemitteln und einer geringeren Konzentration Pfefferminzöl.

Die Evaluation der Patienten erfolgte zu Studienbeginn, nach 4 und nach 8 Wochen durch Bewertung der subjektiven Schmerzen mit einer VAS.

\section{Ergebnisse}

63 Patienten wurden eingeschlossen, 4 beendeten die Studie vorzeitig. Das mittlere Alter betrug 63 Jahre, 54\% waren Frauen.

Die VAS-Werte zeigten eine größere mittlere Schmerzreduktion für die Glukosamin-/Chondroitin-Gruppe (mittlere Veränderung $-3,4 \mathrm{~cm}$, SD $2,6 \mathrm{~cm}$ ) im Vergleich zu der PlaceboGruppe (mittlere Veränderung $-1,6 \mathrm{~cm}$, SD 2,7 cm) nach 8 Wochen (Tab. 1). Somit ergab sich ein Unterschied von $1,8 \mathrm{~cm}$ für die Differenz zwischen den Gruppen (95\%-CI 0,6-2,9 cm; p = 0,002). Dieser signifikante Effekt zeig- te sich bereits nach 4 Wochen $(1,2 \mathrm{~cm}$ mittlere Differenz zwischen den beiden Gruppen, 95\%-CI 0,1-2,4; $\mathrm{p}=0$,03). Patientenaufzeichnungen der VAS-Werte ergaben ab Tag 1 einen im Mittel $1 \mathrm{~cm}$ niedrigeren Wert für die Verum-Gruppe im Vergleich zur Placebo-Gruppe. Nebenwirkungen waren selten und schwach ausgeprägt und in den beiden Gruppen ähnlich verteilt.

\section{Schlussfolgerung}

Die Studie zeigte einen signifikanten Unterschied zwischen den beiden Gruppen nach der Verwendung der Cremes. Die Substanzen Glukosaminsulfat und Chondroitinsulfat in Kombination sind bezüglich Schmerzreduktion bei Kniearthrose gegenüber Placebo signifikant überlegen. Fast alle Patienten erzielten sofortige Schmerzlinderung. Zusätzlich gab es eine größere mittlere nach haltige Reduzierung der Schmerzen bei 4 und 8 Wochen.

\section{Literatur \\ 1 Cohen M, et al.: J Rheumatol 2003;30:523-528.}

Quelle: Vita Health Care AG, Bösingen, Schweiz, www.vita-healthcare.ch.

Tab. 1. Mittelwerte und Standardabweichungen (SD) des VAS Pain Score bei drei Klinikaufenthalten und Veränderungen vom ersten zum dritten Aufenthalt

\begin{tabular}{lllllll}
\hline & \multicolumn{3}{c}{ Mittlerer VAS Pain Score, $\mathrm{cm}(\mathrm{SD})$} & & \multicolumn{2}{l}{$\begin{array}{l}\text { Veränderungen des VAS Pain } \\
\text { Score von Woche 0 zu Woche } 8\end{array}$} \\
\cline { 2 - 3 } \cline { 7 - 8 } & Woche 0 & Woche 4 & Woche 8 & & Mittelwert $(95 \%-C I)$ & SD \\
\hline Placebo $(\mathrm{n}=29)$ & $6,2(1,5)$ & $4,8(2,6)$ & $4,6(2,8)$ & & $-1,6(-2,6$ bis $-0,6)$ & 2,7 \\
Aktiv $(\mathrm{n}=30)$ & $6,1(1,4)$ & $3,5(2,5)$ & $2,8(2,4)$ & & $-3,3(-4,3$ bis $-2,4)$ & 2,6 \\
\hline
\end{tabular}

